\title{
¿Es la atención sanitaria a las personas mayores con enfermedad avanzada una alternativa asistencial de la Medicina Interna?
}

Navarro Sanz $R$. ¿Es la atención sanitaria a las personas mayores con enfermedad avanzada una alternativa asistencial de la Medicina Interna? An Med Interna (Madrid) 2004; 21: 315-316.

El concepto, contenido, limites de actuación, docencia e investigación de la Medicina Interna (MI) está, desde hace años, en continua discusión (1-5). Durante las últimas décadas, se viene hablando de la "crisis de la Medicina Interna" que como señala Ravid (6) significa una cierta inquietud de los profesionales frente una mayor demanda ante el incremento de la edad de la población, mayor presión sobre el control de calidad, mayor responsabilidades, aumento de la cultura social y ambiental de los pacientes, incremento del número de subespecialidades, mayor desarrollo técnico y científico, etc. Esta "crisis" ha sido motivo de constantes debates en los Congresos de las Sociedades de Medicina Interna (3,5). En ellos se valoran de forma positiva los beneficios científicos y asistenciales que aportan dichas especialidades médicas; pero también se ve la excesiva atención al hallazgo local y al detalle técnico con la consiguiente pérdida de la visión unitaria del enfermo que la especialización conlleva.

En numerosos países desarrollados, la MI vuelve a actuar de forma dominante y como "superespecialidad" en aras de una visión del ser humano en su totalidad (3); es decir, de recuperar la visión global del enfermo. Se aprecian una serie de modificaciones en el campo de actuación de la MI debido a cambios de orden social, político y económico que vienen ocurriendo en dichos países [la potenciación de la medicina ambulatoria ante el coste elevado en el hospital; la aparición de nuevas demandas sociales (mejoría de la calidad de vida, rehabilitación, etc.); el aumento de la patología crónica ante la mayor expectativa de vida de la población; los médicos cada vez piensan más en los costes de los tratamientos, en la calidad de vida de los pacientes, etc.)] (5-8).

Teniendo en cuenta la visión unitaria de la MI, el campo de actuación del médico internista, lejos de desmoronarse por la aparición de las especialidades médicas, se ha fortalecido (3). Lo ideal sería que la formación del internista se estableciese en función de las características del trabajo a desempeñar posteriormente, aunque nuestro modelo sanitario no está definido hasta el punto de poder delimitar cómo y dónde van a desarrollar su labor los internistas en el futuro (8). No obstante, aquellos cambios de orden social, político, económico, etc., nos hace presumir que los límites de la práctica de la MI en la actualidad se puedan establecer entre la actividad de la Medicina de Atención Primaria y la de las Especialidades Médicas derivadas de la MI, con una mayor demanda de atención hacía los problemas primarios de salud y la problemática que presentan los pacientes ancianos con pluripatología, con enfermedades crónicas y pacientes en situación clínica de enfermedad terminal (5,9-12). Parece de sentido común buscar un equilibrio entre la MI y las especialidades médicas; así el médico internista puede actuar como consultor de otros especialistas e integrar las opiniones de éstos en beneficio del enfermo. Este equilibrio o quizás una mayor integración se debe buscar, ante la gran demanda de atención sanitaria de las personas mayores con comorbilidad y discapacidad y en situación clínica de enfermedad avanzada, entre la Medicina Geriátrica y la Medicina Paliativa.

La Geriatría nace, como el resto de las especialidades médicas, de la Medicina Interna ante la necesidad de atender los problemas de las personas mayores (13) pero no siempre ha habido una buena relación entre ambas, viviéndose momentos de verdadera aspereza y antagonismo, incluso en países como Inglaterra, cuna de la Geriatría $(14,15)$. Pero actualmente existen suficientes hechos que vienen a demostrar la existencia de un acercamiento mutuo entre las especialidades de Medicina Interna y Geriatría. Así, cada vez más aparecen artículos científicos sobre temas geriátricos en revistas de medicina interna y capítulos de medicina geriátrica en libros de medicina interna, integración de servicios clínicos para el diagnóstico y tratamiento de los problemas de las pacientes ancianos, como por ejemplo en el Reino Unido $(16,17)$ y colaboración entre unidades de Geriatría y servicios de Medicina Interna de hospitales generales (13). Así mismo se reconoce el papel del internista no solo como consultor del paciente geriátrico, sino como "principal" cuidador (18).

La Medicina Paliativa surge como respuesta ante las necesidades de atención sanitaria y apoyo emocional que presentan los pacientes en situación clínica de enfermedad terminal. Se fundamenta en la filosofía del "Movimiento Hospice", que viene determinada por: a) el morir como un fenómeno normal de la vida; b) los síntomas de los pacientes terminales son controlables; y c) la atención a estos pacientes debe ser integral y requie- 
re de un equipo multidisciplinar (19-22). Además la Medicina Paliativa tiene una clara relación con la geriatría, compartiendo puntos en común (21,23): a) el centro de atención es el binomio enfermo-familia; b) defienden un uso adecuado y discriminado de los métodos de diagnóstico y tratamiento con el fin de buscar un adecuado "confort" del paciente; c) el instrumento de la terapéutica es la atención en Equipo Multidisciplinar; y d) son relativamente novedosas (sobre todo en nuestro medio) y cuestionan en ocasiones la metodología y pautas de actuación tradicionales de otras especialidades

El futuro de la Medicina Interna, como señalaba una editorial del Annals of Internal Medicine de diciembre de 1993 (24), se ha venido fundando en una serie de cambios de distinta índole: a) demográficos (envejecimiento de la población, aumento de las enfermedades crónicas en las personas mayores, mayor incidencia de enfermedades degenerativas, conllevan una mayor complejidad de los cuidados y sus problemas médicos deben ser contemplados en conjunto); b) tecnológicos (que van haciendo que los procedimientos diagnósticos y de tratamiento sean cada vez menos invasivos); y c) sociales (cambios a estilos de vida más saludables, acceso universal a los cuidados sanitarios y necesidad de contener el gasto). Estos cambios han venido configurando los cuidados médicos de los tiempos actuales y de las décadas venideras, necesitando de clínicos con base sólida en las especialidades médicas tradicionales y en nuevas disciplinas como epide- miología, medicina del comportamiento, información, análisis de decisión, gestión y ética (25). El internista generalista combina las características de un clínico humanista, diagnosticador, con conocimientos de atención primara, consultor y experto en la prevención de enfermedades, promoción de la salud, cuidados continuados y en el manejo y control de pacientes con enfermedades crónicas y avanzadas (26). Además existen campos de actuación que están en sus inicios y con necesidad de explorar en profundidad, donde la demanda de atención sanitaria de estos grupos de pacientes es primordial y el internista puede y debe tener competencias importantes, como pueden ser las unidades de hospitalización a domicilio, las unidades de corta estancia, unidades de cuidados paliativos, hospitales de crónicos, etc. (27).

Ante todo ello se puede decir que una de las alternativas en el campo de la asistencia, docencia e investigación de la MI puede estar en la atención a las personas mayores con comorbilidad y discapacidad y a los pacientes en situación clínica de enfermedad avanzada.

\section{R. NAVARRO SANZ}

Servicio de Medicina Interna. Hospital Dr. Moliner. Serra. Valencia

\section{Bibliografía}

1. Rozman, C. Reflexiones sobre la Medicina Interna en la Universidad. Med Clin (Barc) 1981; 76: 193-202.

2. Lissen, E. El Departamento de Medicina Interna en las Ciudades Sanitarias de la Seguridad Social. Med. Clin (Barc.) 1983; 80: 167-171.

3. Ortiz Vázquez J. Presente y Futuro de la Medicina Interna como especialidad, como departamento hospitalario y como enseñanza. An Med Interna (Madrid) 1984; 1: 100-107

4. Asenjo Sebastián MA. El Servicio de Medicina Interna en la Organización hospitalaria. Libro de actas del XVIII Congreso de la Sociedad Española de Medicina Interna. 1988; Madrid, 207-226.

5. Lissen E. La medicina interna general a debate: historia de una crisis y su recuperación. Med Clin (Barc) 1995; 105: 142-147

6. Ravid, M. The challenge to internal medicine. Isr J Med Sci 1989; 25: 648-649.

7. López Santolino A. Formación de internistas en Estados Unidos. Libro de actas del XVIII Congreso de la Sociedad Española de Medicina Interna. 1988; Madrid, 29-31.

8. Riancho Moral JA. La formación de internistas en España: educación médica para los próximos 30 años. Libro de actas del XVIII Congreso de la Sociedad Española de Medicina Interna. 1988; Madrid, 67- 70.

9. Freyro JE. Papel del internista en los Centros de Salud. Libro de actas del XVIII. Congreso de la Sociedad Española de Medicina Interna. 1988; Madrid, 71 - 74.

10. Navarro R. Características y necesidades sanitarias y sociales del perfil de pacientes ancianos con enfermedades y dependencias, pacientes con enfermedades de larga evolución y enfermos terminales (PALET). En: Segundo curso de atención socio-sanitaria al paciente anciano, crónico y terminal (PALET). Valencia: Generalitat Valenciana, 1994; 7-17.

11. Jefes de Servicio de Medicina Interna de los Hospitales Comarcales de Catalunya. El futuro de los servicios de medicina interna de los hospitales comarcales de Cataluña: líneas estratégicas en el contexto de cambio en la Sanidad. Med Clin (Barc) 1993; 100: 587-590.

12. American College of Physicians. The role of the future General Internist defined. Ann Intern Med 1994; 121: 616-622.

13. Ribera Casado JM. Medicina Interna versus Geriatría. Especialidades antagónicas o complementarias. An Med Interna (Madrid) 1990; 7: 324326.
14. Ensuring equity and quality of care for elderly people. The interface between geriatic medicine and general internal medicine. A report of the Royal College of Physicians. The Royal College of Physicians of London, 1994.

15. Brocklehurst JC, Davidson C, Moore-Smith B. Interface between geriatric and general medicine. Health Trends 1989; 21:48-50.

16. Grimley Evans J, Graham JM. Medical care of the elderly: Five years on. J Royal Col Phys London 1984; 18 (4): 18-20.

17. Grimley Evans J. Hospital services for elderly people. The United Kingdom experience. En: Grimley Evans J. and Franklin Williams, T, editores. Oxford Textbook of Geriatric Medicine. Oxford: Oxford University Press 1992; 703-706.

18. Hazzard WR, Woolard N. Integrating into the subspecialities of internal medicine. Cancer 1997; 80 (7): 1267-1269.

19. Esle HR. Terminal Care. The national scene and Ihe individual patient. In: The Medical Clinics of North America. Philadelphia: Saunders Company 1982; 1161- 1168 .

20. Doyle D,Hanks GWC, Macdonald N. Introduction. En: D. Doyle. editor. Oxford Textbook of Palliative Medicine. Oxford: Oxford Medical Publications 1993; 3-8.

21. Sanz Ortiz J. Principios y práctica de los cuidados paliativos. Med Clin (Barc) 1989; 92: 143-145.

22. Boletín informativo de la Sociedad Española de Cuidados Paliativos, $\mathbf{n}^{\circ}$ 0, 1992.

23. Núñez Olarte JM, Pérez Almeida E. El enfermo terminal geriátrico en los programas de cuidados paliativos. Geriátrika 1991; 7 : 54-55.

24. What is the future of internal medicine? (editorial). Ann Intern Med 1993; 119: 1144-1145.

25. Jefes de Servicio de Medicina Interna de los Hospitales Comarcales de Cataluña. Evolución de la medicina interna en el marco del sistema sanitario actual. Med Clin (Barc) 1997; 109: 77.

26. Federated Council for Internal Medicine. Generating more generalistics: an agenda ofrenewal for intemal medicine. Ann Intern Med 1993; 119: $1125-1129$.

27. Herrero Huerta F. La Medicina Interna actual. Una visión interesada. Rev Clin Esp 2003; 203: 242-3. 